\title{
The Macroscopic Volume Changes of Selected Polymers Subjected to Uniform Tensile Deformation
}

\author{
JOHN M. POWERS \\ Departments of Mechanical Engineering \\ and Dental Materials \\ and \\ ROBERT M. CADDELL \\ Department of Mechanical Engineering \\ The University of Michigan \\ Ann Arbor, Michigan
}

\begin{abstract}
High density polyethylene (PE), polycarbonate (PC), and polymethylmethacrylate (PMMA) were subjected to uniaxial tensile deformation up to the onset of instability or necking. Simultaneous readings of longitudinal extension and transverse contraction (width and thickness) were obtained continuously during the loading period. From these data, plots of longitudinal versus "average" transverse strain were produced and it was found that the trends were neither constant nor linear over the full strain range employed. Additional plots of per cent volume change versus longitudinal strain indicate that the PMMA and PC show a maximum volume increase of about $0.6 \%$ while $\mathrm{PE}$ shows a maximum volume decrease on the order of $2.5 \%$. Similar volume decreases have been noted by others and it would appear that structural changes are the most likely cause of this behavior.
\end{abstract}

\section{INTRODUCTION}

$I^{n}$ $\mathrm{n}$ recent years there have appeared in the published literature (e.g. 1-4) an increasing number of studies devoted to the cold forming of polymeric solids by traditional metal working processess. If there is to be developed an approach to the cold forming of polymers which parallels that in metal forming plasticity theory, it is essential that further extensive studies be conducted along such lines.

Based upon experimental observation, the yield behavior of metals is taken to be independent of the mean (hydrostatic) stress for any given stress state. This is an extremely fortunate consequence since various yield criteria can be made independent of this "pressure" effect. It would appear that the yield behavior of polymers is influenced by the mean stress which will complicate the situation in comparison with metals. Studies have been conducted in this regard (e.g. 5-7) but at this time there exists no generally accepted yield criterion that could be considered to be reasonably universal.

A second assumption invoked in metal forming analyses pertains to constancy of volume. This too is well supported by experimental observation, and since the entire effort of this present work has to do with studies of volume changes, certain explanations or comments are warranted at this point. In terms of linear elasticity, volume changes are reflected by the magnitude of Poisson's ratio. This "property" is viewed as an elastic constant; often the assumption of a homogeneous, isotropic medium is invoked. In terms of thermodynamic stabilty, the magnitude of this ratio must lie between -1 and +0.5 ; Callen (8) discusses this in detail. A value of 0.5 is equivalent to volume constancy and on this basis, it is often stated that the "plastic" behavior of metals shows a Poisson's ratio of one half.

Rigbi (9) has discussed the major problems connected with viscoelastic effects on Poisson's ratio while Shamov (10) reported on the time dependent behavior of this ratio for "low pressure" polyethylene. Krause et al (11) subjected several polymers to compressive deformations, determined volume changes, then computed average values of Poisson's ratio from the variations in volume. Bonnin et al (12) conducted tensile and shear creep experiments; assuming isotropy they computed values of Poisson's ratio from these other measurements. It should be mentioned that compressive deformations are seldom "uniform" and true isotropy is often likely to be lacking in such materials thus, either of these two approaches $(11,12)$ may be a bit questionable. Hopefully, such problems are avoided in this present 
study since all results are based upon continuous, direct measurements of linear dimensions that show any volume changes and reflect the basic definition of Poisson's ratio.

Benham and McCammond (13) have, in a recent paper, noted that the use of the term "Poisson's ratio" may lead to confusion where the ratio of lateral to longitudinal strain is either non-linear or time dependent. We are in full agreement with that observation since values of that ratio which exceed 0.5 have been reported by several authors; as this "violates" the grounds upon which Poisson's ratio is usually based, another term or name might be more acceptable for such situations. Following the general suggestion of Benham (13), the ratio of lateral contraction to longitudinal extension, $v$, will be called the "contraction ratio" in the remainder of this paper. Where linear, elastic behavior might exist, $v$ would be equivalent to Poisson's ratio, so generally accepted terminology in that regime of deformation is not really by-passed or changed.

\section{EXPERIMENTAL PROCEDURE}

Specimens for uniaxial tensile loading were machined from $3 / 4$ in. diameter, "as-received" rods, obtained from Cadillac Plastics. The test materials were high density polyethylene (PE), polycarbonate (PC), and polymethylmethacrylate (PMMA).

For each material, all test specimens were produced from a single rod in order to reduce possible scatter caused by material variability. One other essential point must be noted here. Since the intent of this study was to investigate one aspect of mechanical behavior of certain polymers using an approach not previously reported, no emphasis was placed upon a detailed description or control of the individual polymers employed. Rather, it was decided deliberately to use commercially supplied materials in this initial investigation. Future studies employing "controlled" variables (e.g. \% crystallinity of PE) are anticipated. Only in this way will a knowledge of the potential range of values of a given polymer, as a function of different "structural" conditions be forthcoming.

Every individual specimen was threaded on the ends for well aligned adaptation to the testing device. The individual gage sections were about $4 \frac{1}{2}$ in. long with a square cross section of $3 / 8$ in. nominal side; these sections were produced on a milling machine. Linear dimensional changes were sensed by a strain gage extensometer (Instron type G-51-12M) while transverse changes across each pair of flat cross section surfaces were sensed by two transverse strain sensors (Instron type G-57-12M). Before testing, these three pickups were carefully calibrated. To provide greater credence in the test results, parallel studies were conducted on two different test setups. On one, the specimens were adapted to a 5 ton Instron machine and the outputs from the three pickups were amplified to drive an XYY recorder. Simultaneously, load readings on the Instron strip chart were correlated with readings on the XYY recorder.
The second setup utilized a $500 \mathrm{KG}$ Instron, the same three pickups but entirely different amplifiers and XYY recorder. It was hoped that the use of different recording equipment by different operators would avoid undue concern about the generality of the test results.

With both setups, the specimens were loaded at the same cross head speed of $0.05 \mathrm{~cm} / \mathrm{min}$ and an individual test was continued until the onset of tensile instability or necking was evident. With the two $\mathrm{Y}$ axes indicating decreases in transverse dimension and the $\mathrm{X}$ axis giving increases in longitudinal dimension, instantaneous values of transverse and longitudinal strain were readily obtainable. In addition, the instantaneous cross sectional measurements were identfied with particular values on the load-extension plot of the strip chart recorder; these were used to provide values of true stress corresponding to values of true or logarithmic strain. All tests were conducted at room temperature (about $25^{\circ} \mathrm{C}$ ).

Because of the number of calculations that were forthcoming, a computer program was written to assist in both accuracy and speed of data reduction. Prior to presenting the results it should be mentioned that at least two tests per material were performed on each of the two setups, the total number for each material being 5 for (PC), 5 for (PMMA), and 7 for $(\mathrm{PE})$.

\section{EXPERIMENTAL RESULTS}

Figures 1-3 portray findings for the test materials; a number of discrete "true" longitudinal-transverse strain combinations produced such curves. For the sake of overall clarity, only the extreme variations for each material are plotted; other test results would fall within each "band". The solid circles and triangles on Figs. 1-3 are not actual test points but are included to identify the extreme variations and to relate them to particular lines plotted on Figs. 4-6. For each discrete "longitudinal" strain employed, the two indivdual "transverse" strain measurements were averaged. Although these two strain measurements often differed, the maximum variation was on the order of $2 \%$ and it seemed that this was most

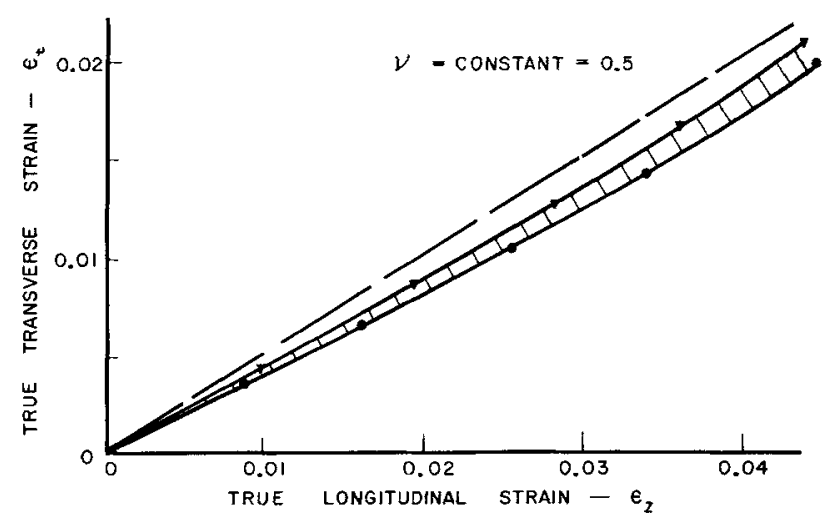

Fig. 1. True transverse versus true longitudinal strain during uniform tensile deformation of $P C$. 


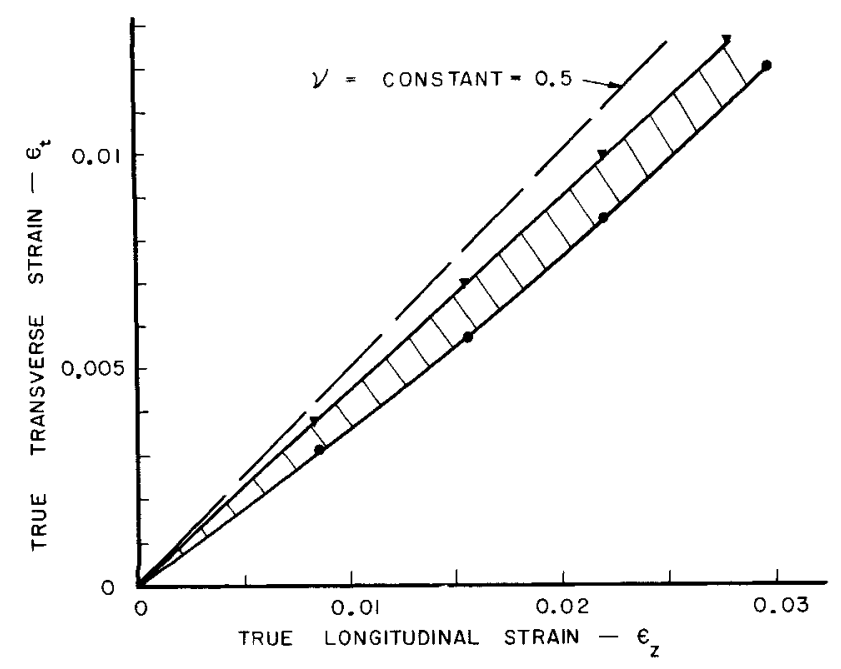

Fig. 2. True transverse versus true longitudinal strain during uniform tensile deformation of PMMA.

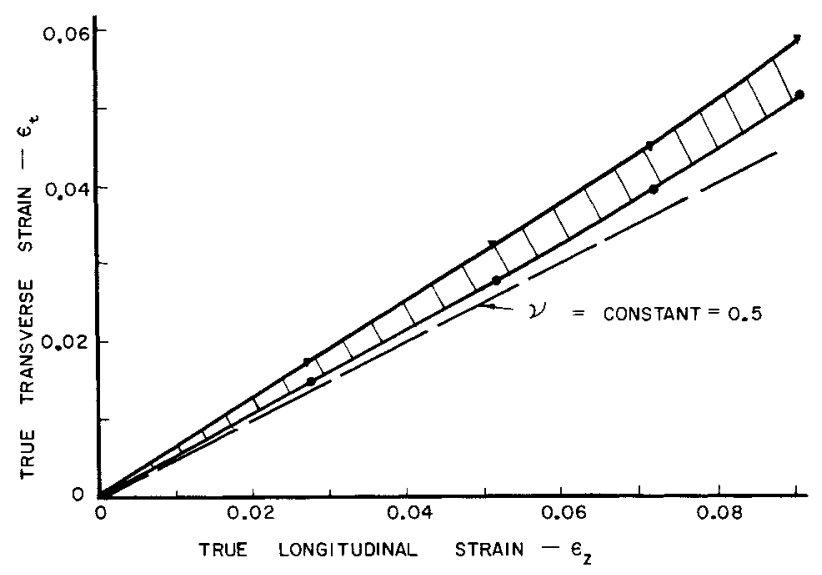

Fig. 3. True transverse versus true longitudinal strain during uniform tensile deformation of high density PE.

likely due to experimental "variations". Note that on each of the first three figures, a dashed straight line (denoted as $v=$ constant $=0.5$ ) is plotted; this serves for comparative purposes only.

Figures 4-6 show the percent volume change plotted against true longitudinal strain. Note that the first two of this group indicated a volume increase whereas the third shows a volume decrease. The "mid points" of the extreme variations per material (i.e. average behavior) have been replotted as single lines on Fig. 7 in order to provide a comparative summary of the three materials.

Figure 8 includes those lines from Figs. 1 and 4 designated by solid circles and shows a means for comparing volume change and transverse strain as functions of longitudinal strain. A detailed discussion on this point is covered in the next section of this paper.

Figure 9 includes the true stress-true strain behavior of these polymers. For each material, the extreme variations encountered fell within the individual cross-hatched band. True stress values were computed by using $\sigma=\frac{L}{A}$ where $L$ is an instantaneous load in pounds and $A$ is the corresponding instantaneous area. The equivalent value of true strain was computed from $\epsilon_{z}=\log _{e}\left(\frac{l}{l_{o}}\right)$ where $l$ is the instantaneous gage length and $l_{o}$ the initial or un-

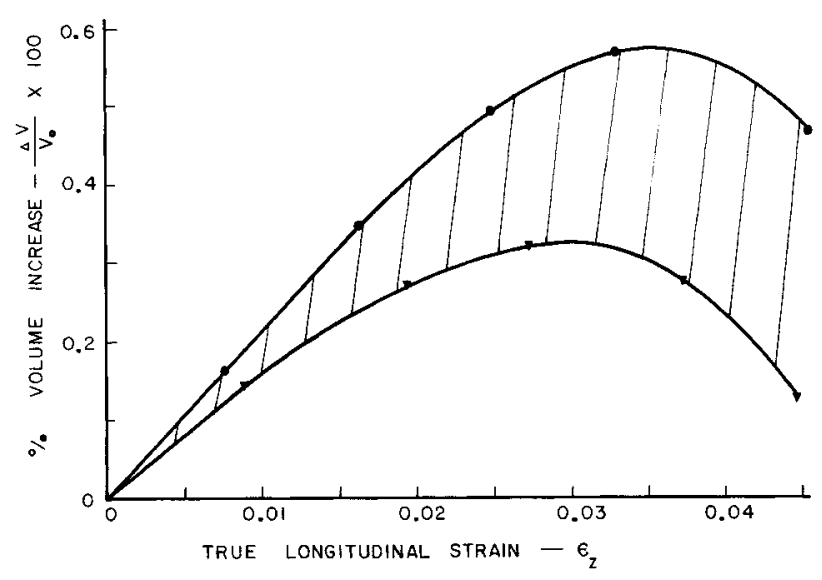

Fig. 4. Percent volume increase of PC as influenced by uniform tensile deformation.

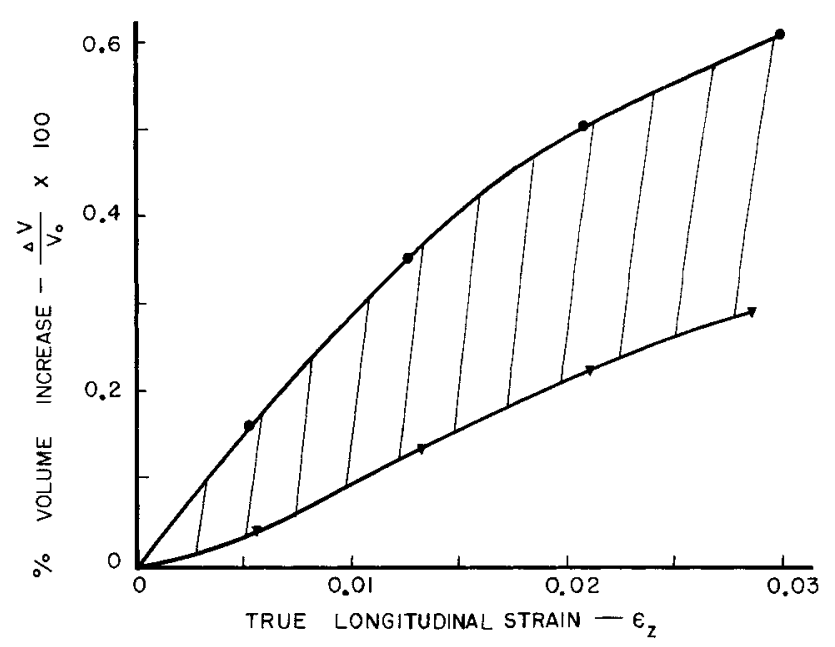

Fig. 5. Percent volume increase of PMMA as influenced by uniform tensile deformation.

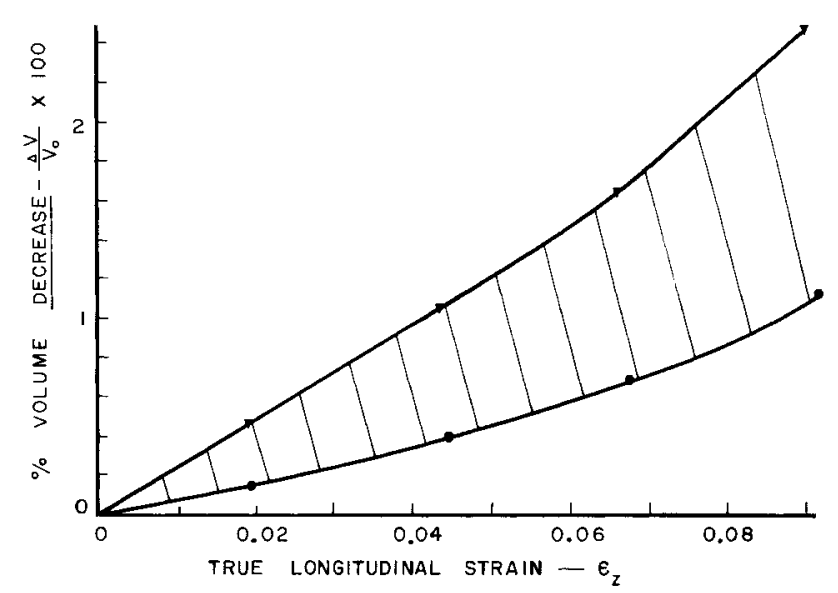

Fig. 6. Percent volume decrease of PE as influenced by uniform tensile deformation. 


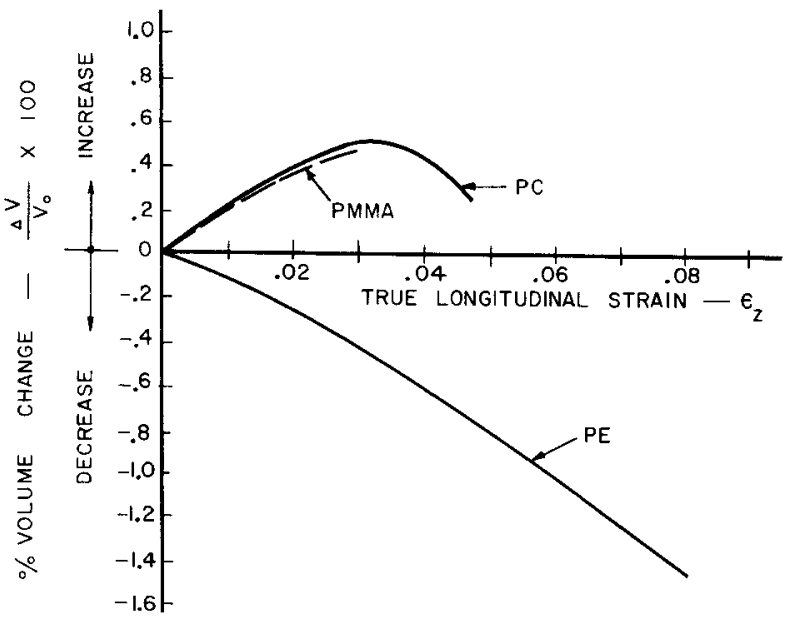

Fig. 7. "Average" percent volume changes of PC, PMMA, and $P E$ as influenced by uniform tensile deformation.

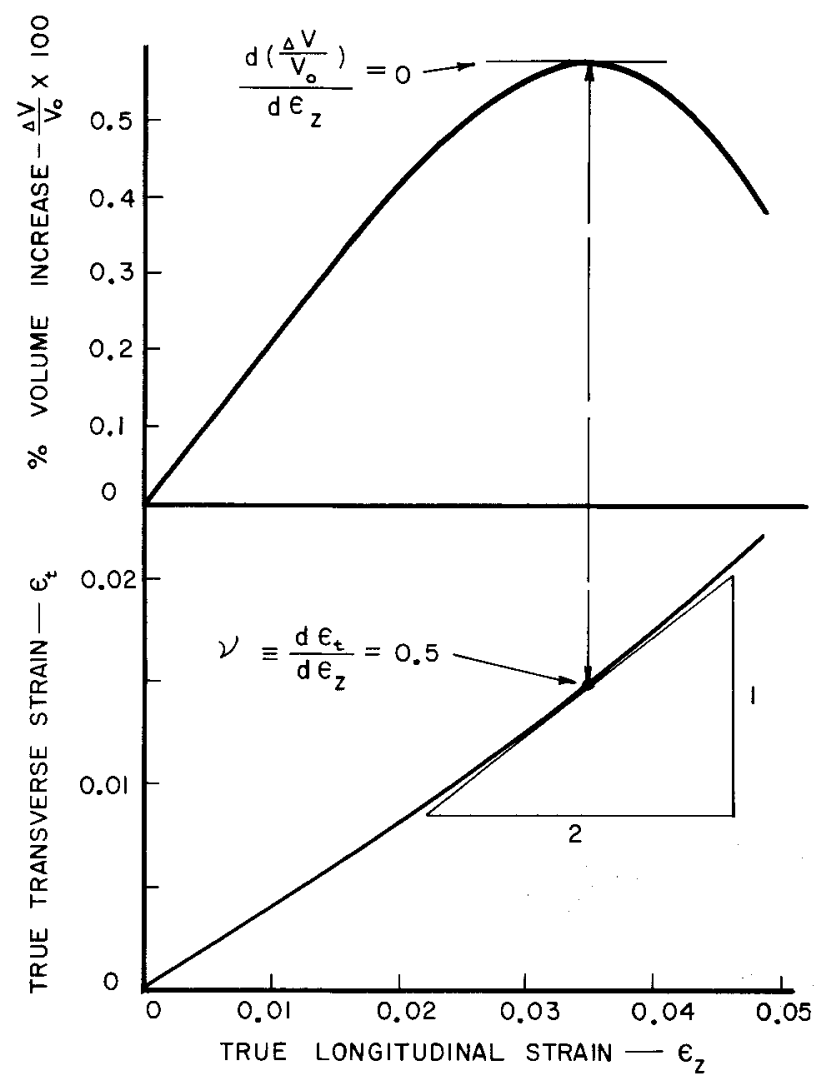

Fig. 8. Illustration of interrelationship of percent volume change and instantaneous value of contraction ratio for one test using PC.

loaded gage length. Note that $\epsilon_{Z} \approx \log _{e}\left(\frac{A_{o}}{A}\right)$ where $A_{o}$ is the initial or unloaded cross sectional area and $A$ the same instantaneous area involved in the computation of true stress. Because the volume is not constant here, $A_{o} l_{o} \neq A l$ although the differences are relatively small.

\section{DISCUSSION}

It can be observed from Figs. 1-3 that the contraction ratio is not a constant for any of these polymers

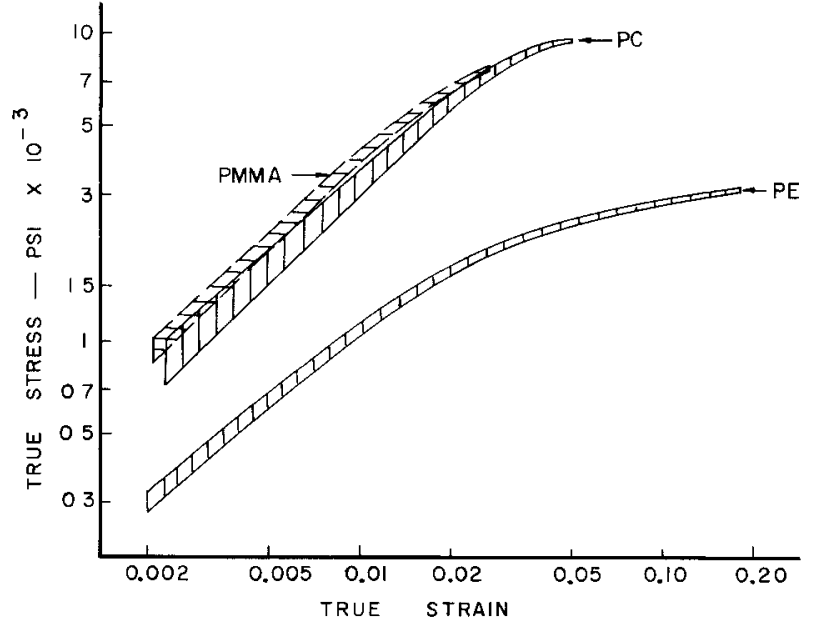

Fig. 9. True stress $(\sigma)$ versus true strain $\left(\epsilon_{Z}\right)$ for $P C, P M M A$, and $P E$ subjected to uniform tensile deformation.

over the strain ranges employed. The nonlinearity of these plots supports that observation, and is in general agreement with recent findings of Darlington and Saunders (14) who used a low density polyethylene in their work.

As a rough approximation, one can fit a straight line to the data obtained in the early stages of each test in order to get an idea of the range of the initial values of the contraction ratio. These are included in Table 1 with corresponding values of longitudinal strain beyond which the behavior becomes exceedingly non-linear. With such behavior, the instantaneous value of the contraction ratio at any total strain $\epsilon_{Z}$, is determined from the slope of the $\epsilon_{Z}$ vs. $\epsilon_{t}$ plot as indicated in Fig. 8. If one defined $v$ in terms of total strains at any point (i.e. a straight line emanating from the origin) this would differ from the "instantaneous" value just mentioned. The results tabulated by Krause (11) demonstrate this quite emphatically.

The results with $\mathrm{PE}$ indicate that a contraction ratio in excess of 0.5 occurs; this implies a volume decrease (or density increase) accompanies tensile deformation. Behavior similar to this has been observed by others $(11,12,13)$ for various materials. Although not fully substantiated at this point, the

Table 1.* Ranges of Measured Properties of the Three Polymers

\begin{tabular}{|c|c|c|c|c|}
\hline \multirow[t]{2}{*}{$\begin{array}{l}\text { Polymer } \\
\text { and } \\
\text { number } \\
\text { of } \\
\text { tests }\end{array}$} & \multicolumn{2}{|c|}{$\begin{array}{l}\text { Contraction ratio } \\
\text { where approxi- } \\
\text { mately linear } \\
\text { over initial } \\
\text { range of } \\
\text { strain }\end{array}$} & \multirow[t]{2}{*}{$\begin{array}{l}\text { Tensile } \\
\text { strength, } \\
S_{u} \text {, in psi } \\
S_{u}=\frac{L_{u}}{A_{o}}\end{array}$} & \multirow[t]{2}{*}{$\begin{array}{c}\text { True strain at } \\
\text { onset of tensile } \\
\text { instability }\end{array}$} \\
\hline & $\nu$ & $\epsilon \mathbf{Z}$ & & \\
\hline $\begin{array}{l}\text { PC-5 } \\
\text { PMMA-5 } \\
\text { PE-7 }\end{array}$ & $\begin{array}{l}0.41-0.44 \\
0.37-0.45 \\
0.54-0.61\end{array}$ & $\begin{array}{l}0.028 \\
0.020 \\
0.070\end{array}$ & $\begin{array}{l}9,050-9,200 \\
7,700-8,500 \\
2,550-2,700\end{array}$ & $\begin{array}{l}0.044-0.049 \\
0.028-0.031 \\
0.095-0.106\end{array}$ \\
\hline
\end{tabular}

* Subscript $u$ refers to conditions at onset of tensile instability while o refers to initial or unloaded condition. 


\section{J. M. Powers and R. M. Caddell}

causes are at present attributed to either possible structural changes $(11,13)$ or anisotropy (12). In particular, Benham (13) has observed this behavior with polypropylene while the present authors in this study, have seen such a result only with polyethylene. Since both of these polymers have a glass transition temperature well below room temperature, whereas most of the others that have been studied have a $T_{g}$ well above room temperature, it is certaintly tempting to suggest that this may be a parameter which forms a dividing line. Further efforts in this regard are obviously needed.

Another point of interest here is the behavior of PC to "peak" and then drop off as induced strain is increased. This implies that beyond the point of peaking, the instantaneous value of the contraction ratio exceeds 0.5 whereas, before that point, the ratio was less than 0.5 . It has been observed that this polymer tends to reach tensile instability (that is the onset of "necking") in a very abrupt manner whereas PE undergoes a very gradual transition before necking becomes evident. (Note that PMMA acts relatively "brittle" compared to the others in that no pronounced neck is formed). The strain at instability and that at which peaking occurs are very similar for PC; although the full physical significance of this is not understood at this time, the coincidence would suggest more than chance.

Perhaps it is of importance to stress that the same general behavior was noted for each group of tests per each polymer so the general trends are felt to be real and not due to any unique feature of the experimental procedure and/or equipment. For completeness and possible reference or comparison, the ranges of tensile strength and instability strain are also included in Table 1 .

\section{CONCLUSIONS}

Within the limits of temperature and strain rate employed in this study, the behavior of PE, PC, and PMMA is generally non-linear in regard to variations in contraction ratio caused by uniaxial tensile loading. A volume decrease of up to $2.5 \%$ has been noted when PE is subjected to tensile loading up to the onset of instability. To date, it appears most likely that structural alterations account for that behavior. With PMMA and PC, volume increases up to $0.6 \%$ were noted although this trend was not constant for the PC for the full strain range employed.

\section{ACKNOWLEDGMENT}

The authors wish to thank Professor A. G. Atkins for his discussions during this study and his suggestion of including a drawing such as Fig. 8.

\section{NOMENCLATURE}

$\epsilon_{t}=$ Average true (logarithmic) transverse strain

$\epsilon_{z}=$ True longitudinal strain $=$

$$
\log _{e}\left(\frac{l}{l_{o}}\right) \approx \log _{e}\left(\frac{A_{o}}{A}\right)
$$

$v=-\epsilon_{t} / \epsilon_{z}=$ Contraction ratio (note that in the usual context of elasticity this is Poisson's ratio)

$\sigma \quad=$ True tensile stress $\equiv L / A$

$L=$ Instantaneous tensile load

$A=$ Instantaneous cross sectional area

$A_{\mathrm{o}}=$ Initial cross sectional area

$l_{o} \quad=$ Initial gage length

$l=$ Instantaneous gage length

$V_{o}=$ Initial volume of gage section $=A_{o} l_{o}$

$\Delta V=$ Change in initial volume during tensile deformation

\section{REFERENCES}

1. A. Buckley and H. A. Long, Polym. Eng. Sci., 9, 664 (1969).

2. L. J. Broutman and R. S. Patil, Technical Papers, 28th Annual Technical Conference, Society Plastic Engineers, p. 20, New York, N. Y. (May 1970).

3. L. J. Broutman and S. Kalpakjian, S.P.E. J., 25, 46 (1969).

4. C. S. Lee, R. M. Caddell, and G. S. Y. Yeh, Matl. Sci. Eng., 10, 235 (1972).

5. W. Whitney and R. D. Andrews, J. Poly. Sci., C, 16, 2981 (1967).

6. S. S. Sternstein, R. Ongchin, and A. Silverman, Appl. Pol. Symp., 7, 175 (1968).

7. S. S. Sternstein and L. Ongchin, A.C.S. Pol. Prep., 10, 1117 ( 1969)

8. H. B. Callen, "Thermodynamics," p. 213, John Wiley and Sons (1960).

9. Z. Rigbi, "High Speed Testing, Vol VI, The Rheology of Solids," Interscience (1967).

10. I. V. Shamov, Mekahnika Polimerov, 1, 52 (1965).

11. I. Krause, A. J. Segreto, and H. Przirembel, Mat. Sci. and Eng., 1, 239 (1966).

12. M. J. Bonnin, C. M. R. Dunn, and S. Turner, Plast. \& Pol., Journ. Plast. Inst., p. 517 (1969).

13. P. B. Benham and D. McCammond, Plast. and Pol., Jour. Plast Inst., 39, 130 (1971).

14. M. W. Darlington and D. W. Saunders, J. Macrom. Sci., B5, 2, (1971). 